\title{
Establishment of a Rapid Detection Method for Rice Blast Fungus Based on One-Step Loop-Mediated Isothermal Amplification (LAMP)
}

\author{
Ling Li, Shu Ya Zhang, and Chuan-Qing Zhang ${ }^{\dagger}$ \\ The Key Laboratory for Quality Improvement of Agricultural Products of Zhejiang Province, School of Agricultural and Food \\ Sciences, Zhejiang Agriculture and Forest University, Hangzhou 311300, China
}

\begin{abstract}
Rice blast is one of the most serious diseases for rice, and controlling the filamentous fungus Magnaporthe oryzae that causes rice blast is crucial for global food security. Typically, early infected rice does not show symptoms. Therefore, the early diagnosis of rice blast is particularly important to avoid uncontrollable propagation of rice blast fungus. In the present work, a rapid and efficient loop-mediated isothermal amplification (LAMP) method was developed to detect the pathogen at the early infected stage of rice. The Alb1 superfamily hypothetical protein MGG_04322, a nuclear shuttling factor involved in ribosome and melanin biogenesis, was chosen as the target for designing the LAMP primers. The LAMP assay enabled rapid detection of as little as $10 \mathrm{pg}$ of pure genomic DNA of $M$. oryzae. In addition, we established the quantitative LAMP (q-LAMP) detection system to quantify the conidia of

rice blast fungus. The q-LAMP assay enabled rapid detection (within $35 \mathrm{~min}$ ) of the fungal spores at a sensitivity of 3.2 spores $/ \mathrm{ml}$. In addition, the assay sets up the linearization formula of the standard curve as $y=0.3066+15.33 x$ (where $x=$ amplification of time), inferring that spore number $=10^{0.60 y}$. In addition, the q-LAMP assay was successfully used to detect the presence of the virulence strains of $M$. oryzae (wild type) in comparison with that of the two mutant strains by quantifying the biomass within host tissue. These results provide a useful and convenient tool for detecting $M$. oryzae that could be applied in the incubation period of rice blast before symptoms appear.

Keywords: loop-mediated isothermal amplification (LAMP), Magnaporthe oryzae, MGG_04322, q-LAMP, rice blast
\end{abstract}

Rice is a very important food crop because it feeds a majority of the world's population (Delseny et al. 2001). As the most devastating disease in all rice growing areas, rice blast destroys a rice crop equivalent to that needed to feed 60 million people (Manandhar et al. 1998; Talbot 2003). The hemibiotrophic fungus Magnaporthe oryzae (T. T. Hebert) M. E. Barr (anamorph: Pyricularia oryzae Cavara) is the causal agent of rice blast (Ou 1985). Because of the experimental tractability and economic importance of $M$. oryzae, it is usually used as a model organism for the investigation of fungi-oriented plant diseases (Ebbole 2007). At all of the development stages of rice plants, either the leaves, stems, roots, nodes, or panicles can be attacked by the rice blast fungus.

Although breeding disease-resistant varieties is the main way to prevent and control rice blast, host resistance is lost and rapidly surpassed by variation in $M$. oryzae, which results in resistant cultivars that are ineffective in 2 to 3 years. Herein, to solve this problem affecting food security, alternative strategies, especially early diagnosis, are urgently needed to harness the infectivity of $M$. oryzae.

Early diagnosis of rice blast can avoid uncontrolled propagation of pathogens, and is of utmost importance in controlling the wide spread of the pathogen, preventing direct and indirect economic losses such as the use of unnecessary fungicides. As a result, the toxic substances can be reduced in the environment. Numerous isothermal amplification techniques have been established in the past decades for pathogen identification at the early stage of infection. Due to nucleic acid amplification, detection is highly specific and sensitive, and it is much faster to identify pathogens than morphological identification

${ }^{\dagger}$ Corresponding author: C. Zhang; cqzhang@zafu.edu.cn

Funding: This work is supported by Zhejiang Key Research and Development Program (2015C02G2010084 and 2018C02G2011099) and the Natural Science Foundation of Zhejiang Province (LQ17C010001).

The author(s) declare no conflict of interest.

Accepted for publication 7 March 2019.

() 2019 The American Phytopathological Society using microscopy (Boonham et al. 2008; Parida et al. 2008). The loop-mediated isothermal amplification (LAMP) was first designed to amplify a limited amount of DNA copies into a million copies within an hour without the need for thermal cycling (Notomi et al. 2000). Thus, LAMP is well suited for diagnostic use and facilitates the potential identification of remote locations even at the initial infection stage. The LAMP product can be visualized by means of gel electrophoresis, magnesium pyrophosphate precipitation, and turbidimetric and colorimetric reactions using a color-changing reagent such as hydroxyl naphthol blue (Goto et al. 2009; Mori et al. 2004). LAMP assays have been successfully developed to detect fungi such as Fusarium graminearum, Aspergillus carbonarius, and Sclerotinia sclerotiorum (Duan et al. 2014; Niessen and Vogel 2010; Storari et al. 2013). Currently, a quantitative LAMP (q-LAMP) assay with additional reagents and more complex equipment allows LAMP to be performed in a quantitative real-time format, and it was developed to detect plant pathogens such as Apple chlorotic leaf spot virus, Candida glabrata, and Vibrio nigripulchritudo in shrimp (Fall et al. 2011; Peng et al. 2017). Recently, to quantify the airborne inoculum of the Lolium pathotype in turf field plots, q-LAMP with a spore trap system has been utilized in the United States (Villari et al. 2017). This system can identify the inoculated turfgrass plots with as few as 10 conidia up to 12 days before symptoms developed (Villari et al. 2017). Such reports are sufficient to illustrate that the development of a q-LAMP assay to detect rice blast would be efficient and valuable.

In this study, LAMP assays have been developed for rapid, easy, specific, and highly sensitive detection in the early infected stage of $M$. oryzae. To our knowledge, this is the first protocol developed for the detection of $M$. oryzae using both a LAMP assay and qLAMP assay. Hopefully, the method established here will allow the fast identification of the potential risks caused by $M$. oryzae in rice crops.

\section{Materials and Methods}

Fungal cultures and DNA extraction. The wild-type strain Guy11 (Notteghem and Silue 1992) and the mutants $\Delta$ mopex $14 / 17$ and $\Delta$ mopex 19 (Li et al. 2014, 2017) of M. oryzae were donated by Dr. Jiaoyu Wang (Zhejiang Academy of Agricultural Sciences). The mutant strains $\triangle M o P E X 14 / 17$ and $\triangle M o P E X 19$ are obtained 
by knocking out MoPEX14/17 and MoPEX19, respectively, from the wild-type strain Guy11. Moreover, the pathogenicity of $\triangle M o P E X 14 /$ 17 is reduced significantly compared with Guy11, and that of $\triangle M o P E X 19$ is lost completely. The other strains were saved by our laboratory. All of the strains were cultured routinely on complete medium (CM) at $28^{\circ} \mathrm{C}$ for 3 to 14 days (Talbot et al. 1993).

For DNA extraction, each strain was grown in CM lipid medium on a rotary shaker $(100 \mathrm{rpm})$ for 3 days at $28^{\circ} \mathrm{C}$. Through filtration, each fungus mycelium was collected on filter paper. For conidia DNA extraction, conidia were washed from the 10-day-old CM plate colonial surface with sterilized water and a small brush, filtered with three-layer lens-cleaning paper, counted on a hemocytometer under a

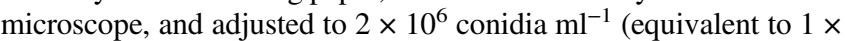
$10^{4}$ conidia per $5 \mu \mathrm{l}$ ). For conidia DNA from leaf material extraction, the conidia of $\Delta$ mopex 14/17, $\Delta$ mopex 19 , and Guy11 were harvested from the CM plate and cultivated at $28^{\circ} \mathrm{C}$ for 10 days, adjusted to $1 \times$ $10^{5}$ conidia liter $^{-1}$, and inoculated on barley leaves. The inoculated leaves were sampled at $6,12,24,36$, and $48 \mathrm{~h}$ postinoculation (hpi).The total genomic DNA was obtained using the cetyltrimethylammonium bromide (CTAB) method (Doyle 1991). Each isolated DNA concentration was determined by agarose gel electrophoresis and measured by Nanodrop 2000 (Thermo Fisher). All samples used for DNA extraction are listed in Table 1.

LAMP and q-LAMP reactions. The MGG_04322 sequence was used to design specific LAMP primers for M. oryzae. The MGG_ 04322 gene plays an important role in ribosome and dihydroxynaphthalene (DHN)-melanin biosynthesis, which is necessary for pathogenesis (Lundqvist et al. 1994; Tsai et al. 1998). MGG_04322 gene as the highly specific target, was designed for the LAMP primers to detect $M$. oryzae The LAMP primers contain forward inner primer (FIP), backward inner primer (BIP), and two outer (F3 and B3) primers and were designed using the Primer Explorer V4 software program (http://primerexplorer.jp), based on the MGG_04322 gene sequence (Notomi et al. 2000). The best primer selection was based on $\Delta \mathrm{G}$ values of less than or equal to $-4 \mathrm{Kcal} / \mathrm{mol}$ at the $3^{\prime}$ end of F3/B3 and F2/B2 and $5^{\prime}$ ends of F1c and B1c.

LAMP reactions were performed using the above-described primer sets (Fig. 1; Table 2). Each 25- $\mu$ l reaction volume contained primers FIP and BIP at $0.25 \mu \mathrm{M}$ liter $^{-1}$, primers F3 and B3 at $0.2 \mu \mathrm{M}$ liter $^{-1}$, betaine at $1.0 \mathrm{mM}_{\text {liter }}{ }^{-1}$, dNTPs (Takara Bio Inc.) at $2.0 \mathrm{mM} \mathrm{liter}^{-1}$, Tris$\mathrm{HCl}(\mathrm{pH} 8.8)$ at $25 \mathrm{mM}$ liter $^{-1}, \mathrm{KCl}$ at $12.5 \mathrm{mM}$ liter $^{-1},\left(\mathrm{NH}_{4}\right)_{2} \mathrm{SO}_{4}$ at $12.5 \mathrm{mM} \mathrm{liter}^{-1}, \mathrm{MgCl}_{2}$ at $10 \mathrm{mM} \mathrm{liter}{ }^{-1}, 0.125 \%$ (vol/vol) Triton X100, Bst DNA polymerase ((New England Biolabs) at $0.2 \mathrm{U}_{\text {liter }}{ }^{-1}$, hydroxynaphthol blue (HNB; Sigma-Aldrich) at $150 \mu \mathrm{M} \mathrm{liter}{ }^{-1}$, and $1 \mu l$ of the target DNA sample extracted as described.

Table 1. Fungal isolates used during the current study and respective amplification results in loop-mediated isothermal amplification (LAMP) assays

\begin{tabular}{|c|c|c|c|}
\hline Number & Species & Isolate $^{\mathbf{a}}$ & Results \\
\hline 1 & Magnaporthe oryzae (wild-type) & Guy11 & + \\
\hline 2 & M. oryzae (mutant) & $\Delta$ торех14/17 & + \\
\hline 3 & M. oryzae (mutant) & Amopex19 & + \\
\hline 4 & Fusarium fujikuroi & CGMCC 3.1108 & - \\
\hline 5 & Penicillium citrinum & ACCC 31507 & - \\
\hline 6 & Ustilaginoidea virens & ACCC 2711 & - \\
\hline 7 & Alternaria alternata & ACCC 36843 & - \\
\hline 8 & Rhizoctonia solani & ACCC 36246 & - \\
\hline 9 & Botrytis cinerea & B05.10 & - \\
\hline 10 & M. oryzae (field strain) & M. $o-1$ & + \\
\hline 11 & M. oryzae (field strain) & M. $o-2$ & + \\
\hline 12 & M. oryzae (field strain) & M. $o-3$ & + \\
\hline 13 & M. oryzae (field strain) & M. $o-4$ & + \\
\hline 14 & M. oryzae (field strain) & M. o-5 & + \\
\hline 15 & M. oryzae (field strain) & M. $o-6$ & + \\
\hline 16 & M. oryzae (field strain) & M. $o-7$ & + \\
\hline 17 & M. oryzae (field strain) & M. $o-8$ & + \\
\hline
\end{tabular}

${ }^{\text {a }}$ CGMCC $=$ China General Microbiological Culture Collection Center and ACCC $=$ Agricultural Culture Collection of China .
Most of the q-LAMP amplification reactions components were consistent with LAMP above, expect for $1 \mu$ l of the target DNA sample replaced with $5 \mu$ l of template DNA (a conidium initial concentration of $1 \times 10^{4}$ conidia per $5 \mu$ l extracted by CTAB), and added $1 \times$ SYBR Premix Ex TAQ (Takara Bio), and the volume was also adjusted to $25 \mu \mathrm{l}$ with nucleic acid-free water. The protocol was performed as follows: a cycle of $30 \mathrm{~s}$ at $95^{\circ} \mathrm{C}, 40$ cycles of $15 \mathrm{~s}$ at $95^{\circ} \mathrm{C}$ and $1 \mathrm{~min}$ at $60^{\circ} \mathrm{C}$, then followed by signal collection. Quantitative polymerase chain reaction (q-PCR) amplification and detection were performed in a Bio-Rad CFX96 Q-LAMP detection system (Bio-Rad Laboratories).

Optimization of LAMP and q-LAMP reaction conditions. The LAMP reaction mixtures were incubated for $45 \mathrm{~min}$ at 61,62 , 63,64 , or $65^{\circ} \mathrm{C}$ to determine the optimal reaction temperature. The reaction mixtures were incubated at an optimal temperature of $64^{\circ} \mathrm{C}$ and amplified $45 \mathrm{~min}$ in a heated block, then incubated at $4^{\circ} \mathrm{C}$ for $15 \mathrm{~min}$ to terminate the reactions. The visual color change in HNB (from violet to sky blue) was examined to judge the reaction results; $1 \%$ agarose gel electrophoresis was used to further check the reaction extent. The q-LAMP reaction mixtures (with or without $M$. oryzae DNA as template) were incubated for $80 \mathrm{~min}$ at $61.8,62.1$, $62.6,63.4,64.4,65.2,65.6$, or $66^{\circ} \mathrm{C}$ to determine the optimal reaction temperature.

Specificity of the LAMP and q-LAMP. To verify the LAMP and q-LAMP specificity, the assay results were compared with the DNA of $M$. oryzae and of six other plant-pathogenic fungi (i.e., $F$. fujikuroi, Penicillium citrinum, Ustilaginoidea virens, Alternaria alternata, Rhizoctonia solani, and Botrytis cinerea) and the negative control used nuclease-free water. In particular, the LAMP assay was conducted at $64^{\circ} \mathrm{C}$ for $45 \mathrm{~min}$ as described previously. With the coassessment of HNB-visualized color change and gel electrophoresis, the reaction results were rigorously discriminated. All of the fungal samples were treated in triplicate, and the experiment was performed three times.

LAMP and q-LAMP sensitivity. Template DNA from $M$. oryzae was progressively diluted from 10 to $10^{-6} \mu \mathrm{g} \mu \mathrm{l}^{-1}$. LAMP (with HNB) assays were then performed using the obtained samples. Specifically, the LAMP products were visualized after the reactions and the products were measured using gel electrophoresis. Three replications were set for each treatment group, and three duplicates were performed for each sample. To test the sensitivity of the q-LAMP assay, serial dilutions of $M$. oryzae conidia fivefold serially diluted (from $10^{4}$ to 1.6 conidia $\mathrm{ml}^{-1}$ ) were examined separately.

Evaluation of the LAMP assay using rice leaves. Rice leaves with symptoms of rice blast were collected from five fields in Zhejiang Province, China, in 2017. The five samples were subjected to LAMP. When LAMP reactions were finished, they were assessed based on color change and based on gel electrophoresis.

Inoculation of M. oryzae and the q-LAMP detection. We then tested the availability of using q-LAMP to monitor and relatively quantify the development of $M$. oryzae in vivo by comparing the biomass of the fungus in infected host plants. Mopex14/17 and Mopex 19 are two genes involved in the peroxisomal biogenesis of $M$. oryzae and are required in the pathogenicity of the fungus. The previous inoculation test and microscopy detection proved that infection of the mutant of $\Delta$ mopex 19 was fully inactive, while that of $\Delta$ mopex $14 / 17$ was reduced greatly (Li et al. 2014, 2017). As the same procedures (Li et al. 2014), the wild-type strain Guy11, $\Delta$ mopex14/ 17 , and $\Delta$ mopex 19 conidia were harvested from 10-day-old CM cultures. The obtained strains were then resuspended at $1 \times 10^{5}$ conidia $\mathrm{ml}^{-1}$ with $0.25 \%$ (wt/vol) gelatin (Talbot et al. 1993). Then, $20 \mu \mathrm{l}$ of spore suspension was placed on detached leaf segments of 7-day-old barley ZJ-8, incubated at $28^{\circ} \mathrm{C}$ in darkness for $24 \mathrm{~h}$, and subsequently in light for $24 \mathrm{~h}$. Specific samples were taken at 6, 12, 24, 36, and $48 \mathrm{hpi}$, then subjected to q-LAMP detection.

\section{Results}

Design of LAMP primers. The LAMP primers (Fig. 1; Table 2) for $M$. oryzae were selected by using the open-access Primer Explorer V4 and checked by comparison with all available relevant 
sequences (Fig. 1). The primers were chosen to allow specific amplification of $M$. oryzae and did not show any similarities to other sequences available in the National Center for Biotechnology Information (NCBI) GenBank database. During the design of the LAMP primers, the $\Delta \mathrm{G}$ values of the $3^{\prime}$ ends of the F3, B3, F2, and $\mathrm{B} 2$ primers and $5^{\prime}$ ends of the F1c and B1c primers were determined, and the values were $-4.6,-4.8,-4.9,-5.4,-5.5$, and -5.6 $\mathrm{Kcal} / \mathrm{mole}$. All $\Delta \mathrm{G}$ values were less than $-4 \mathrm{Kcal} / \mathrm{mol}$. According to the nucleotide search tool (BLAST) in the NCBI sequence database, the targeting of the MGG_04322 primers was checked and had high specificity. Finally, a set of four primers was selected based on their high species specificity and sensitivity targeting the MGG_ 04322 sequence of M. oryzae.

Optimization and development of the LAMP assay. The LAMP assay was positive only for M. oryzae (Guy11, M.o-1, M.o-2, M.o-3, M.o-4, M.o-5, M.o-7, and M.o-8), and no positive DNA products were observed when another six fungi ( $F$. fujikuroi, $P$. citrinum, $U$. virens, A. alternata, $R$. solani, and $B$. cinerea) were used as templates. A positive reaction resulted in the production of a typical ladder-like electrophoresis pattern (Fig. 2B). At the same time, a color variation in the reaction solutions from violet to azure could be observed by the naked eye (Fig. 2A). In contrast, the negative reaction did not show any color variation. Hence, the typical electrophoresis pattern was absent. Pure cultures were used to extract the template DNA, and the minimum detection limit for M. oryzae could

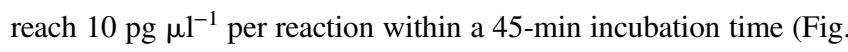
$3 \mathrm{~A}$ and $\mathrm{B})$.

Evaluation of the LAMP assay using infected leaves. To evaluate the efficiency of the LAMP assay in detecting M. oryzae within infected leaves, rice blast leaves collected from four different fields of Jinhua, Zhejiang Province, China in 2017 were tested by the LAMP assay. Nuclease-free water was set as the negative control, while the conidium of $M$. oryzae was set as the positive control. The infected samples resulted in the production of a typical ladderlike electrophoresis pattern (Fig. 4B), accompanied by a color variation in the reaction mix from violet to azure (Fig. 4A).

Development and evaluation of q-LAMP. To efficiently detect rice blast fungus, a q-LAMP assay was then developed and evaluated. The optimal q-LAMP reaction time and temperature were first determined. We set eight temperature gradients from 61.8 to $66^{\circ} \mathrm{C}$ $\left(61.8,62.1,62.6,63.4,64.4,65.2,65.6\right.$, and $\left.66^{\circ} \mathrm{C}\right)$; the optimum reaction temperature was $63.4^{\circ} \mathrm{C}$, and positive results were obtained at 35 min (Fig. 5A). The specificity of the LAMP primer pair MGG_ 04322 was also tested in a q-LAMP assay using genomic DNA from $M$. oryzae and the other six isolates (F. fujikuroi, P. citrinum, $U$. virens, A. alternata, $R$. solani, and $B$.cinerea) (Table 1$)$. The test results showed that no amplification product was observed with genomic DNA isolated from the other six plant-pathogenic fungi and the negative control (Fig. 5B). The sensitivity of the q-LAMP assay and the conidia DNA were determined by testing serially diluted (fivefold

ATGGCCAAGAAAGCACCGTCGAAACACTCCCGAGCGGCGCGCCGGGCGACCTCGCCCAGCATCAACACG

GACAAGTCGCTCAAGGAGGTGCGGCTGCCGGAGCAGTCGATAAACCAGCGACCGGCCGTGCTGGCCGCA

CACCACCACGCTGGCGTCTCCAAGAAGCAAAAGCACCGCAAGAGCCATATGACGTCCAAGATGCGCCGAC

GCCAGGAGAAGAGCATGGACCGCGCCGAGGCTGTCATGGACCGGACGGCCAAGAAGACGGAGAAGAGC ABL1-F3 ABL1-F2

TTCGGCCAGGCCAAGACGGTCGAGGGCAGGAGGAAGGCTTGGGACGACATCAACGCCCAGGCTCTGGG ABL1-F1C

GGCGGAGAAGGGGGAGAAGAAGTCCAAGAAGCAGCTCGAGGCCGAGGCGGAGCAGGCAGAGGTCGA ABL1-B1C ABL1-B2

CAAGTTCTTTGCAAAGGAAGACGATGATGCGGCCATGGGCAACGCTCCGGTCGACGGGGTCGTGTCAAAA ABL1-B3

TTCGATGCGATTTCTGAAAGTGCGCAACCAGCAACAACAGCAACAAGCGACGATGACGAAATGTTGTGA

Fig. 1. A forward inner primer (FIP) consisted of the complementary sequence of F1c and F2, and a backward inner primer (BIP) consisted of B1c and B2. Outer primers F3 and B3 are required for initiation of the loop-mediated isothermal amplification (LAMP) reaction.

Table 2. List of loop-mediated isothermal amplification primers used for detection of Magnaporthe oryzae in this studya

\begin{tabular}{llc}
\hline Primer name & \multicolumn{1}{c}{$\mathbf{5}^{\prime}$ to 3' Oligonucleotide sequence } & Length (bp) \\
\hline F3 & GCCAGGCCAAGACGGT & 16 \\
B3 & GCGCACTTTCAGAAATCGC & 19 \\
FIP (F1c-F2) & GGCCTCGAGCTGCTTCTTGGACGGAAGGCTTGGGACGACA & 41 \\
BIP (B1c-B2) & AAGGAAGACGATGATGCGGCCCGAATTTTGACACGACCCCG & 42 \\
\hline
\end{tabular}

${ }^{\text {a }}$ Forward inner primer (FIP) is a hybrid primer consisting of the F1c sequence and the F2 sequence and backward inner primer (BIP) is a hybrid primer consisting of the B1c sequence and the B2 sequence. 
from $1 \times 10^{4}$ conidia $\mathrm{ml}^{-1}$ ) samples of $M$. oryzae that were quantified via a blood-counting chamber. The results showed that the sensitivity number of the newly established q-LAMP assay was $3.2{\text { conidia } \mathrm{ml}^{-1}}^{-1}$ (Fig. 5C). There was a good linear correlation between the value of $\log ^{10}$ (six gradient dilution conidia number of $M$. oryzae) and the threshold time. The q-LAMP assay standard curve $\left(R^{2}=0.95\right)$ fit well with a log-linear curve. The linearization formula of the standard
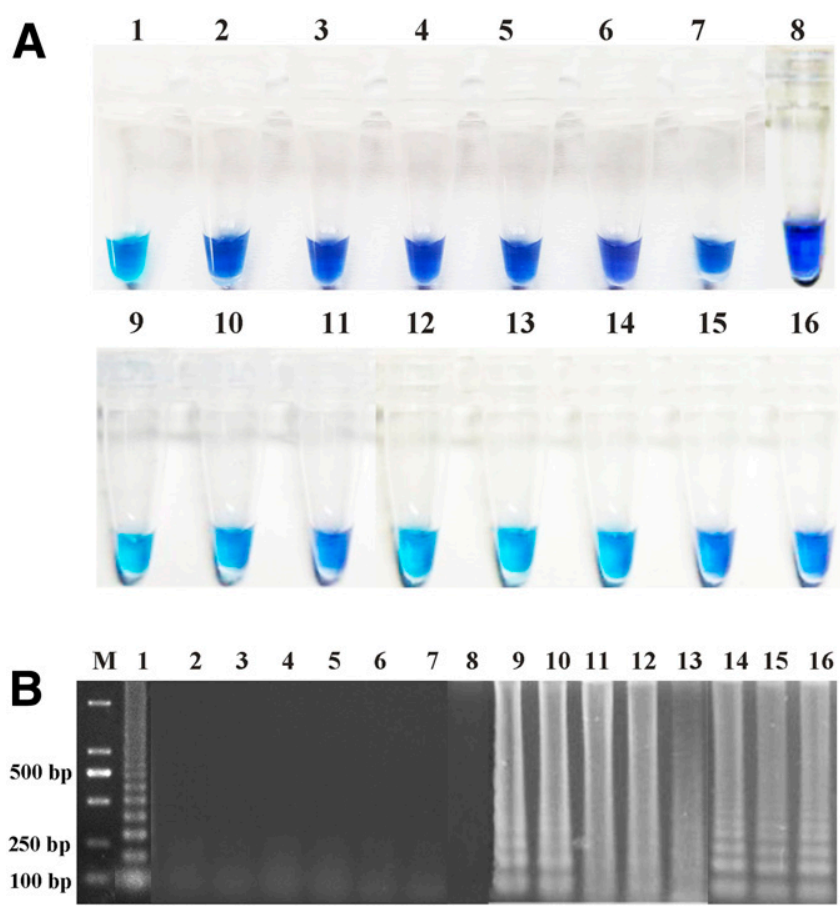

Fig. 2. Specificity of the loop-mediated isothermal amplification (LAMP) detection of Magnaporthe oryzae. Assessment based on A, hydroxynaphthol blue visualization of color change or B, agarose gel electrophoresis analysis of the LAMP products. Lane M, Maker; lane 1, M. oryzae; lane 2, Fusarium fujikuroi; lane 3, Penicillium citrinum; lane 4, Ustilaginoidea virens; lane 5, Alternaria alternata; lane 6, Rhizoctonia solani; lane 7, Botrytis cinerea; lane 8, double-distilled $\mathrm{H}_{2} \mathrm{O}$; lane $9, \mathrm{M}$. $0-1$; lane 10, M. o-2; lane 11, M. o-3; lane 12, M. o-4; lane 13, M. o-5; lane 14, $M$. $0-6$; lane 15, M. o-7; and lane 16, M. o-8.

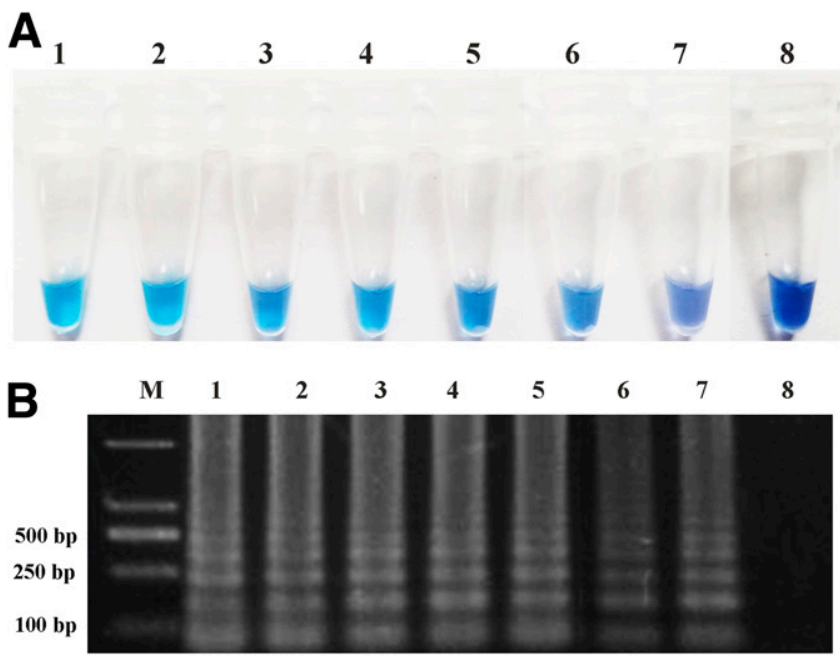

Fig. 3. Sensitivity of loop-mediated isothermal amplification (LAMP) for detection of Magnaporthe oryzae genomic DNA. Detection by A, LAMP and hydroxynaphthol blue visualization, B, LAMP and gel electrophoresis. Concentrations of template DNA $\left(\mu g \mu l^{-1}\right)$ per reaction were lane $1=10$, lane $2=10^{0}$, lane $3=10^{-1}$, lane $4=$ $10^{-2}$, lane $5=10^{-3}$, lane $6=10^{-4}$, lane $7=10^{-5}$, and lane $8=10^{-6}$. Lane $\mathrm{M}$ indicates a 100-bp ladder. curve was $y=0.3066+15.33 x$ (where $x=$ amplification of time), thus inferring spore number $=10^{0.60 y}$ (Fig. 5D).

Comparing the biomass within the host tissue of different $M$. oryzae virulence strains by q-LAMP. We detected and compared the biomass of the wild-type strain Guy11 and the MoPEX14/17 and MoPEX19 deletion mutants at different time points postinoculation. No positive q-LAMP results were obtained before 6 hpi from leaves inoculated with either Guy11 or the two mutants. At 12 hpi, although no visible symptom was found on any incubated leaves, positive q-LAMP signals were detected on samples inoculated with Guy11 and $\Delta$ mopex14/17, with the threshold time at $38.07 \pm 0.24$ and $51.32 \pm 1.25$, respectively. At $24 \mathrm{~h}$, the threshold times of Guy11 and $\Delta$ mopex $14 / 17$ obtained from the q-LAMP detection were $23.14 \pm 0.34$ and $30.90 \pm 0.55$, respectively. At this time point, the initial symptoms appeared on Guy11-inoculated leaves, whereas the symptoms on $\Delta$ mopex $14 / 17$ - and $\Delta$ mopex 19 -inoculated leaves were still invisible. At $36 \mathrm{hpi}$, the q-LAMP detection produced threshold times at $20.47 \pm 0.28$ and $28.02 \pm 0.38$ for Guy11 and $\Delta$ mopex $14 / 17$, respectively, and the visible lesions appeared on the leaves inoculated with Guy11 and as well those with $\Delta$ mopex14/ 17. Until $48 \mathrm{hpi}$, the threshold times of Guy11 and $\Delta$ mopex $14 / 17$ were $19.14 \pm 0.20$ and $25.64 \pm 0.61$, respectively, whereas that of $\Delta$ mopex19 still undetectable (Fig. 6). Thus, the signals and time thresholds reflected development of the fungus within host tissue and quantified relatively the infection ability of different strains. In addition, the signals were detectable before there were symptoms, which corresponded well with the severity of symptoms for different strains.

\section{Discussion}

It is well known that $M$. oryzae-related rice blast is the major disease impeding the cultivation and, hence, the output of rice crops. To resolve this problem, the rapid and accurate detection of M. oryzae in different species or strains is necessary for clarifying the pathogenic factors and underlying mechanisms. In addition, related disease management strategies can then be constructed. For the sake of disease surveillance or intervention procedures, fast and accurate detection of an adequately low level of pathogen is critical to diagnose rice blast at early infection times. In practice, a timely detection of $M$. ory$z a e$ at the early infection stage would facilitate the formulation of proper decisions to control this plant disease. With the development of the LAMP technique, it became possible to implement fast detection and accurate identification of $M$. oryzae infections.

Nevertheless, there also exist many restrictive factors that prevent LAMP technology from detecting rice blast fungus and being successfully applied and promoted in the field. One should know that, with the existence of potential environmental contaminants inhibiting polymerase activity, as well as the random loss of assay sensitivity, the LAMP assay for field quantification of fungal pathogens may not be as feasible as expected. Another factor limiting the sensitivity was primer redesign. In particular, previous studies on LAMP assays mainly used primers targeting the housekeeping gene internal transcribed spacer or elongation factor 1- $\alpha$. At the same time, some specific transposable element sequences such as Pot2, MGR583, and MoTeR have also been utilized to identify M. oryzae (Farman 2002; Pieck et al. 2017). In our study, an Alb1 superfamily hypothetical protein, MGG_04322, a nuclear shuttling factor involved in ribosome and DHN-melanin biogenesis, was chosen to specifically detect $M$. oryzae. Consequently, the LAMP assay developed here used four primers: F3, B3, FIP, and BIP. To verify the efficiency and specificity of the four primers, we used the DNA extracted from $M$. oryzae and from six other important plant-pathogenic fungi as templates for the LAMP assay. The LAMP assay correctly distinguished $M$. oryzae from the other pathogens. Therefore, one can assert that the primers and LAMP assay devised here are specific for M. oryzae.

Typically, the limiting step for high-throughput trials is the preparation of high-quality DNA from fungi, which is usually time and labor consuming (Chi et al. 2009). In addition, it also requires expensive and sophisticated equipment. The extraction of fungal genomic 
DNA generally contains two major steps: (i) the breaking of cell walls and (ii) the extraction and purification of genomic DNA (Zhang et al. 2010). In most situations, liquid nitrogen or glass rods are used to break down cell walls (Zhang et al. 2010). Then, CTAB extraction buffer is often used to extract the genomic DNA (Doyle 1991), and the obtained samples can be purified through phenol/chloroform extraction and isopropanol/ethanol precipitation (Ashktorab and Cohen 1992). Even though DNA with satisfactory quantity and quality could be obtained through these techniques, these procedures are tedious and time consuming. To improve the efficiency of DNA extraction techniques, abundant methods have been implemented, and some effective ones have been developed (Al-Samarrai and Schmid 2000; González-Mendoza et al. 2010; Griffin et al. 2002). However, these methods are still relatively inappropriate for LAMP detection in the field.

The LAMP assays developed in this work established a simple, fast, and inexpensive diagnostic method and q-LAMP as a quick and accurate quantitative method for the early detection of the

A

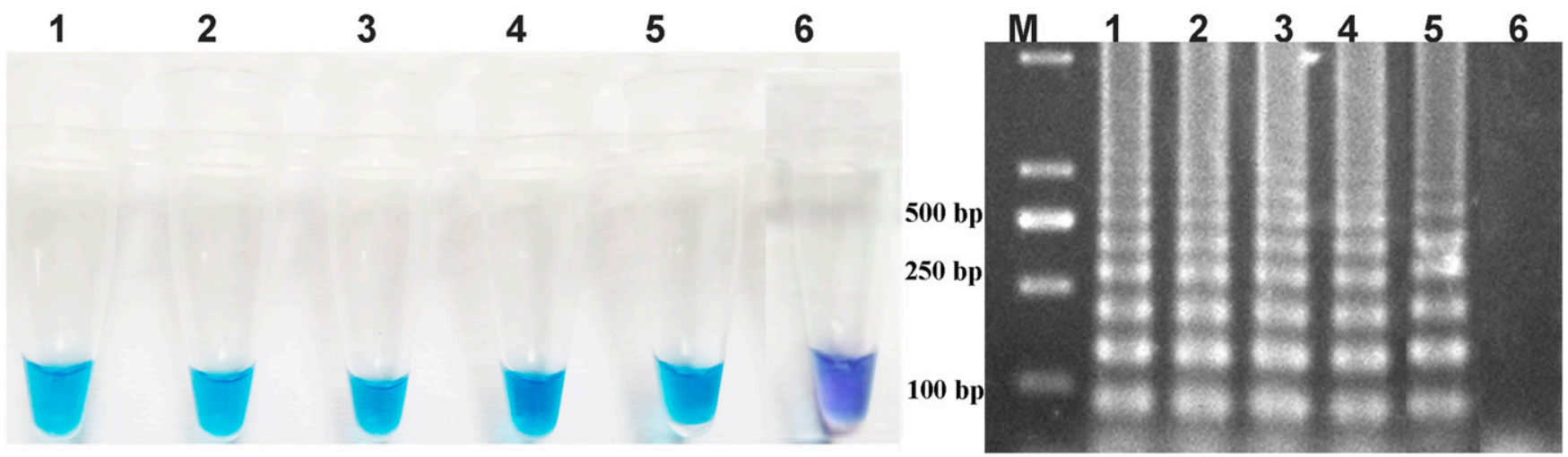

Fig. 4. Evaluation of the loop-mediated isothermal amplification (LAMP) assay using infected leaves. Detection by A, LAMP and hydroxynaphthol blue visualization and B, LAMP and gel electrophoresis. Lane M, Maker; lane 1, Magnaporthe oryzae DNA as the positive control; lanes 2 to 5 , four rice blast leaves from different area of Jinhua, Zhejiang Province, China in 2017; lane 6, nuclease-free water as the negative control.
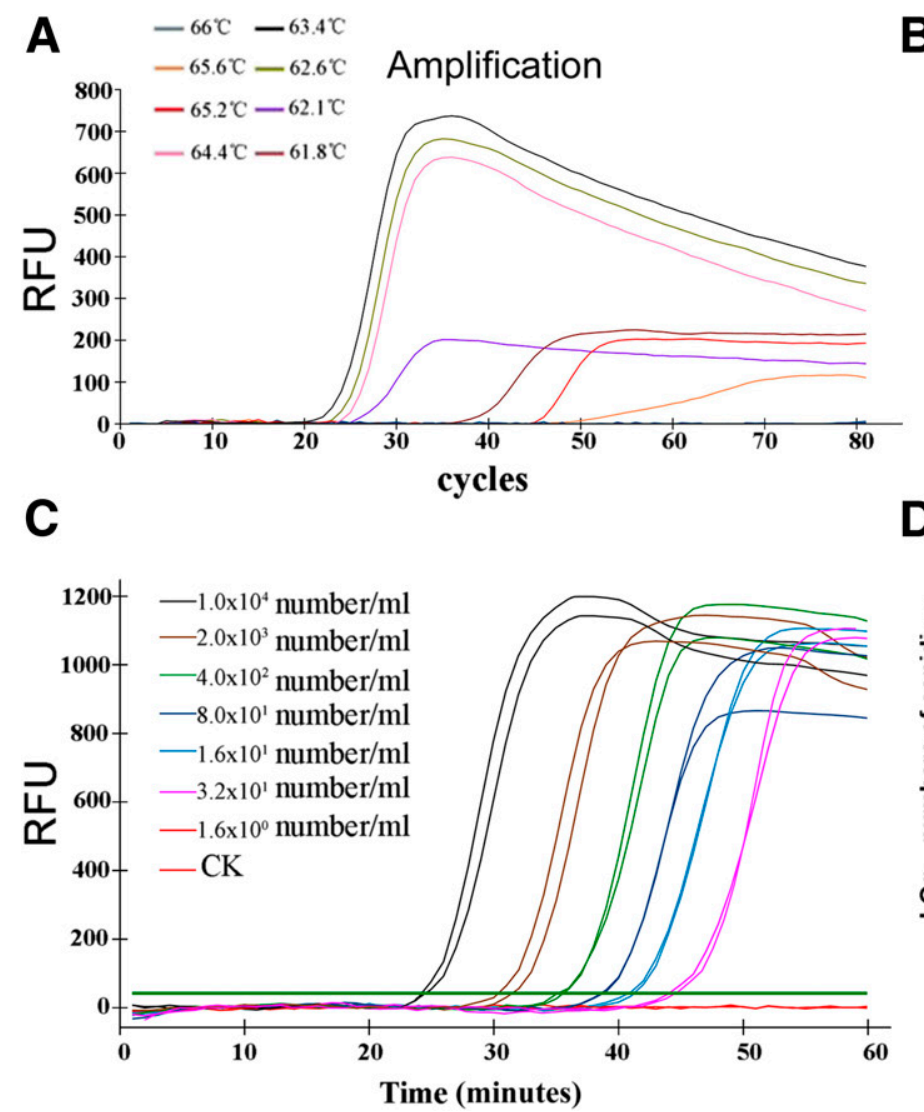

B
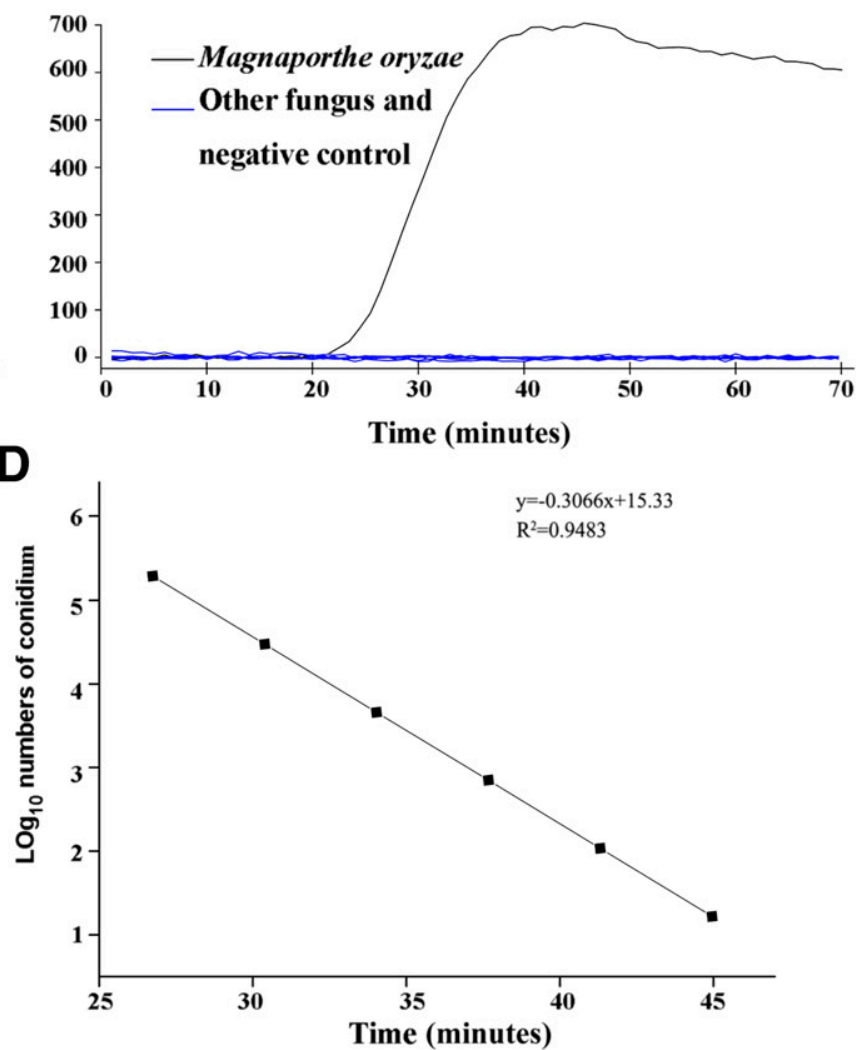

Fig. 5. Establishment of the quantitative loop-mediated isothermal amplification (q-LAMP) assay. A, Optimization of the LAMP reaction temperature. Temperature set up was 61.8, $62.1,62.6,63.4,64.4,65.2,65.6$, and $66^{\circ} \mathrm{C}$ to determine the optimal reaction temperature. RFU = relative fluorescence unit. B, Specificity of the q-LAMP assay for Magnaporthe oryzae. Six fungi were amplified by the assay, including M. oryzae, F. fujikuroi, Penicillium citrinum, Ustilaginoidea virens, Alternaria alternata, Rhizoctonia solani, and Botrytis cinerea. Nuclease-free water was used as a negative control. The assay was repeated three times. C, Sensitivity of the q-LAMP assay for $M$. oryzae. ABL1 genes were tested in the serial dilutions of $M$. oryzae conidium. Nuclease-free water was used as a negative control (CK). The assay was repeated three times. D, q-LAMP assays of serial dilutions of template DNA of $M$. oryzae. Times plotted against the $\log ^{10} \mathrm{CFU} \mathrm{\textrm {ml } ^ { - 1 }}$ derived from the DNA of $M$. oryzae; three repetitions for each dilution are indicated. 
pathogen $M$. oryzae on rice leaves. It is noteworthy that reliable results were obtained by direct LAMP and q-LAMP amplification of $M$. oryzae conidia, and no fungal isolation step was involved. Furthermore, the direct observation of in-tube color change simplifies the unnecessary handling of tubes after the LAMP reaction. The optimized procedure can substantially reduce the possibility of crosscontamination between samples. This procedure enables a simple and clear positive or negative reading of reaction results, which can be carried out by nonqualified staff even without the use of UV illumination equipment.

According to the optimized LAMP assay, it is possible to identify $M$. oryzae rapidly (in approximately $35 \mathrm{~min}$ ) with a much lower detection limit than conventional PCR amplification. Moreover, qLAMP can be implemented without a loss of time due to thermal cycling, and this has rendered the amplification process extremely efficient in comparison with conventional q-PCR. The results showed that, by adding very low amounts of DNA to the LAMP or q-LAMP master mix, it was still sufficient to detect the target DNA from M. oryzae. According to previous studies, the sensitivity of LAMP was 100 or even 1,000 times higher than that of PCR (Duan et al. 2014; Zhou et al. 2014). Our research revealed that LAMP

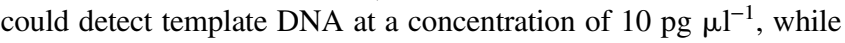
other reports suggested that the detection limit of LAMP was 10 to

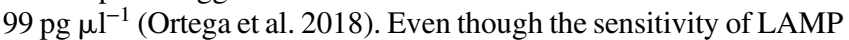
in this study was not as high as that shown in other studies, the sensitivity was still high enough for detection. In addition, it was reported that, with the help of a spore strap system, a q-LAMP assay was able to detect as few as 10 conidia from a Lolium pathotype for up to 12 days before symptoms (Villari et al. 2017). In the present study, we established q-LAMP to detect the sensitivity of the newly assay,

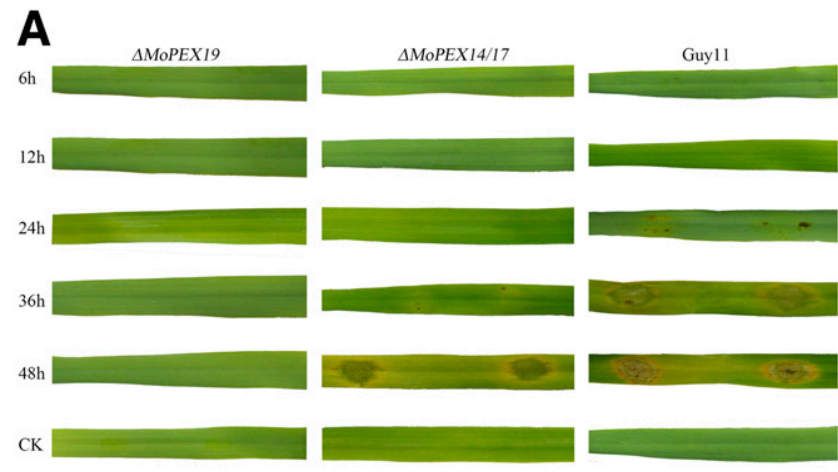

\section{B}

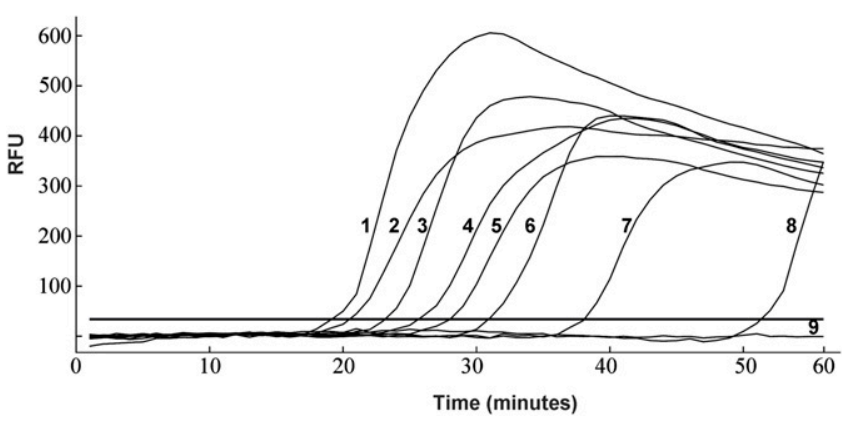

Fig. 6. Quantitative loop-mediated isothermal amplification (q-LAMP) assay for comparing the virulence of Magnaporthe oryzae strains by quantifying the biomass within the host tissue. A, Pathogenicity test of $\Delta$ mopex14/17, $\Delta$ mopex19, and wildtype Guy11. B, Quantitative determination of $M$. oryzae in leaf tissue by q-LAMP. $\mathrm{RFU}=$ relative fluorescence unit. Amplification curves $1,2,3$, and 7 represent the leaves that were inoculated with the wild-type strain Guy11 conidium suspension for $48,36,24$, and $12 \mathrm{~h}$, respectively; the amplification curves $4,5,6$, and 8 represent the leaves that were inoculated with strain $\Delta$ mopex14/17 conidium suspension for $48,36,24$, and 12 h, respectively; and amplification curve 9 represents the leaves that were inoculated with strain $\Delta$ mopex 19 conidium suspension for $48,36,24,12$, and $6 \mathrm{~h}$, strain Guy11 and $\Delta$ mopex $14 / 17$ conidium suspension for $6 \mathrm{~h}$, and the negative control. which was 3.2 conidia $\mathrm{ml}^{-1}$. In addition, there was a good linear correlation between the value of $\log ^{10}$ and the threshold time. The linearization formula of the standard curve was $y=0.3066+15.33 x$ (where $x=$ amplification of time); thus, it could be inferred that spore number $=10^{0.60 y}$. Therefore, q-LAMP can be used to quantitatively detect the conidia of rice blast fungus in the field at different growth periods, and the results will provide a reference for prevention and treatment of rice blast. A q-LAMP assay was developed as a mediated isothermal amplification assay to detect Erysiphe necator in the field. In the early testing of the assay, sensitivity was one spore. However, by the end of the trial, it decreased to more than 20 spores, and the cause was not determined (Thiessen et al. 2018). We plan to use q-LAMP to detect the conidia of rice blast fungus in the field.

As shown by the results, the utility of the LAMP method for $M$. oryzae detection was further confirmed by determining diseased rice leaves from four different rice fields in Jinhua, Zhejiang Province, China. As expected, the detection efficiency was significantly improved by applying the newly developed LAMP assay. Therefore, the LAMP assay may be used for the detection of M. oryzae in plants, which merits further exploration. As a filamentous fungus-specific peroxin, Mopex 14/17 plays a key role in importing the peroxisomal matrix proteins and endows $M$. oryzae with full virulence. In addition, another protein named MoPex 19 also participates in the maintenance of peroxisomal structure and Woronin bodies, holding the key for normal metabolism and development in the rice blast fungus. Thus, compared with the wild-type strain Guy11, the reduced virulence of M. oryzae is accompanied by the deletion of MoPEX14/ 17 , and the pathogenicity decreased with the diminishment of MoPEX19 (Li et al. 2014, 2017). In our study, the q-LAMP assay was successfully used to detect the presence of the virulence of $M$. oryzae strains (wild type) in comparison with that of the two mutant strains by quantifying the biomass within host tissue. We tested the availability of using q-LAMP to monitor and relatively quantify the development of $M$. oryzae in vivo by comparing the biomass of the fungus in infected host plants. The q-LAMP, as an inexpensive, grower-conducted monitoring technology, may provide sufficient information about inoculum presence and concentration for producers to apply targeted fungicide applications.

Currently, chemical treatment is still the principal way to control rice blast. Furthermore, LAMP can also be developed to rapidly detect resistant isolates regulated by different fungicide mechanisms. Due to its low cost, high simplicity, efficiency, and specificity, the LAMP method will open the door to rapid detection, especially in the monitoring of succinate dehydrogenase inhibitor resistance or other in-field and high-throughput experiments (Fan et al. 2018). For instance, a highly sensitive LAMP assay was applied to rationalize a decision-making process and implement the timing of fungicide operations (Thiessen et al. 2016, 2017).

In conclusion, this is the first assay developed for the detection of $M$. oryzae using both LAMP and q-LAMP assays at the early infection stage. The newly developed LAMP assay can specifically detect low levels of $M$. oryzae, and the q-LAMP assay can be used for precise quantification.

\section{Acknowledgments}

We thank J. Wang (Zhejiang Academy of Agricultural Sciences) for providing the wild-type Guy11, $\Delta$ mopex $14 / 17$, and $\Delta$ mopex 19 strains.

\section{Literature Cited}

Al-Samarrai, T. H., and Schmid, J. 2000. A simple method for extraction of fungal genomic DNA. Lett. Appl. Microbiol. 30:53-56.

Ashktorab, H., and Cohen, R. J. 1992. Facile isolation of genomic DNA from filamentous fungi. Biotechniques 13:198-200.

Boonham, N., Glover, R., Tomlinson, J., and Mumford, R. 2008. Exploiting generic platform technologies for the detection and identification of plant pathogens. Eur. J. Plant Pathol. 121:355-363.

Chi, M. H., Park, S. Y., and Lee, Y. H. 2009. A quick and safe method for fungal DNA extraction. Plant Pathol. J. 25:108-111.

Delseny, M., Salses, J., Cooke, R., Sallaud, C., Regad, F., Lagoda, P., Guiderdoni, E., Ventelon, M., Brugidou, C., and Ghesquière, A. 2001. Rice genomics: Present and future. Plant Physiol. Biochem. 39:323-334. 
Doyle, J. 1991. DNA protocols for plants. Pages 283-293 in: Molecular Techniques in Taxonomy. G. M. Hewitt, A. W. B. Johnston, and J. P. W. Young, eds. Springer-Verlag, Berlin, Germany.

Duan, Y., Ge, C., Zhang, X., Wang, J., and Zhou, M. 2014. A rapid detection method for the plant pathogen Sclerotinia sclerotiorum based on loopmediated isothermal amplification (LAMP). Australas. Plant Pathol. 43:61-66.

Ebbole, D. J. 2007. Magnaporthe as a model for understanding host-pathogen interactions. Annu. Rev. Phytopathol. 45:437-456.

Fall, J., Chakraborty, G., Kono, T., Maeda, M., Suzuki, Y., Itami, T., and Sakai, M. 2011. Quantitative loop-mediated isothermal amplification method for the detection of Vibrio nigripulchritudo in shrimp. Fish. Sci. 77:129-134.

Fan, F., Yin, W., Li, G., Lin, Y., and Luo, C. 2018. Development of a LAMP method for detecting SDHI fungicide resistance in Botrytis cinerea. Plant Dis. 102:1612-1618.

Farman, M. L. 2002. Pyricularia grisea isolates causing gray leaf spot on perennial ryegrass (Lolium perenne) in the United States: Relationship to P. grisea isolates from other host plants. [Erratum: Nov 2002, v. 92 (11), p. 1253.] Phytopathology 92:245-254.

González-Mendoza, D., Argumedo-Delira, R., Morales-Trejo, A., Pulido-Herrera, A., Cervantes-Díaz, L., Grimaldo-Juarez, O., and Alarcón, A. 2010. A rapid method for isolation of total DNA from pathogenic filamentous plant fungi. Genet. Mol. Res. 9:162-166.

Goto, K., Uchida, H., Sasa, M., and Ando, M. 2009. Emitting direction control apparatus for LAMP. United States Patent No. 7511440B2.

Griffin, D. W., Kellogg, C. A., Peak, K. K., and Shinn, E. A. 2002. A rapid and efficient assay for extracting DNA from fungi. Lett. Appl. Microbiol. 34: 210-214.

Li, L., Wang, J., Chen, H., Chai, R., Zhang, Z., Mao, X., Qiu, H., Jiang, H., Wang, Y., and Sun, G. 2017. Pex14/17, a filamentous fungus-specific peroxin, is required for the import of peroxisomal matrix proteins and full virulence of Magnaporthe oryzae. Mol. Plant Pathol. 18:1238-1252.

Li, L., Wang, J., Zhang, Z., Wang, Y., Liu, M., Jiang, H., Chai, R., Mao, X., Qiu, H., and Liu, F. 2014. MoPex19, which is essential for maintenance of peroxisomal structure and Woronin bodies, is required for metabolism and development in the rice blast fungus. PLoS One 9:e85252.

Lundqvist, T., Rice, J., Hodge, C. N., Basarab, G. S., Pierce, J., and Lindqvist, Y. 1994. Crystal structure of scytalone dehydratase-A disease determinant of the rice pathogen, Magnaporthe grisea. Structure 2:937-944.

Manandhar, H. K., Lyngs Jørgensen, H. J., Mathur, S. B., and SmedegaardPetersen, V. 1998. Suppression of rice blast by preinoculation with avirulent Pyricularia oryzae and the nonrice pathogen Bipolaris sorokiniana. Phytopathology 88:735-739.

Mori, Y., Kitao, M., Tomita, N., and Notomi, T. 2004. Real-time turbidimetry of LAMP reaction for quantifying template DNA. J. Biochem. Biophys. Methods 59:145-157.

Niessen, L., and Vogel, R. F. 2010. Detection of Fusarium graminearum DNA using a loop-mediated isothermal amplification (LAMP) assay. Int. J. Food Microbiol. 140:183-191.

Notomi, T., Okayama, H., Masubuchi, H., Yonekawa, T., Watanabe, K., Amino, N., and Hase, T. 2000. Loop-mediated isothermal amplification of DNA. Nucleic Acids Res. 28:e63.
Notteghem, J. L., and Silue, D. 1992. Distribution of the mating type alleles in Magnaporthe grisea populations pathogenic on rice. Phytopathology 82: 421-424.

Ortega, S.F., Tomlinson, J., Hodgetts, J., Spadaro, D., Gullino, M. L., and Boonham, N. 2018. Development of loop-mediated isothermal amplification assays for the detection of seedborne fungal pathogens, Fusarium fujikuroi and Magnaporthe oryzae, in rice seed. Plant Dis. 102:1549-1558.

Ou, S. H. 1985. Rice Diseases. Commonwealth Mycological Institute, Kew, Surrey, U.K

Parida, M., Sannarangaiah, S., Dash, P. K., Rao, P. V., and Morita, K. 2008. Loop mediated isothermal amplification (LAMP): A new generation of innovative gene amplification technique; perspectives in clinical diagnosis of infectious diseases. Rev. Med. Virol. 18:407-421.

Peng, D., Xie, J., Qiang, W., Ling, K. S., Guo, L., Fan, Z., and Zhou, T. 2017. Onestep reverse transcription loop-mediated isothermal amplification assay for detection of Apple chlorotic leaf spot virus. J. Virol. Methods 248:154-158.

Pieck, M. L., Ruck, A., Farman, M. L., Peterson, G. L., Stack, J. P., Valent, B., and Pedley, K. F. 2017. Genomics-based marker discovery and diagnostic assay development for wheat blast. Plant Dis. 101:103-109.

Storari, M., Von, R. R., Pertot, I., Gessler, C., and Broggini, G. A. 2013. Identification of ochratoxin A producing Aspergillus carbonarius and A. niger clade isolated from grapes using the loop-mediated isothermal amplification (LAMP) reaction. J. Appl. Microbiol. 114:1193-1200.

Talbot, N. J. 2003. On the trail of a cereal killer: Exploring the biology of Magnaporthe grisea. Annu. Rev. Microbiol. 57:177-202.

Talbot, N. J., Ebbole, D. J., and Hamer, J. E. 1993. Identification and characterization of MPG1, a gene involved in pathogenicity from the rice blast fungus Magnaporthe grisea. Plant Cell 5:1575-1590.

Thiessen, L. D., Keune, J. A., Neill, T. M., Turechek, W. W., Grove, G. G., and Mahaffee, W. F. 2016. Development of a grower-conducted inoculum detection assay for management of grape powdery mildew. Plant Pathol. 65: 238-249.

Thiessen, L. D., Neill, T. M., and Mahaffee, W. F. 2017. Timing fungicide application intervals based on airborne Erysiphe necator concentrations. Plant Dis. 101:1246-1252.

Thiessen, L. D., Neill, T. M., and Mahaffee, W. F. 2018. Development of a quantitative loop-mediated isothermal amplification assay for the field detection of Erysiphe necator. PeerJ 6:e4639.

Tsai, H. F., Chang, Y. C., Washburn, R. G., Wheeler, M. H., and Kwonchung, K. J. 1998. The Developmentally regulated alb1 gene of Aspergillus fumigatus: Its role in modulation of conidial morphology and virulence. J. Bacteriol. 180: 3031.

Villari, C., Mahaffee, W. F., Mitchell, T. K., Pedley, K. F., Pieck, M. L., and Hand, F. P. 2017. Early detection of airborne inoculum of Magnaporthe oryzae in turfgrass fields using a quantitative LAMP assay. Plant Dis. 101:170-177.

Zhang, Y. J., Zhang, S., Liu, X. Z., Wen, H. A., and Wang, M. 2010. A simple method of genomic DNA extraction suitable for analysis of bulk fungal strains. Lett. Appl. Microbiol. 51:114-118.

Zhou, D., Guo, J., Xu, L., Gao, S., Lin, Q., Wu, Q., Wu, L., and Que, Y. 2014. Establishment and application of a loop-mediated isothermal amplification (LAMP) system for detection of cry1Ac transgenic sugarcane. Sci. Rep. 4:4912. 